Review

\title{
Service Delivery Innovation in Health Care for Patient Satisfaction at Primary and Secondary Level
}

\author{
${ }^{1}$ Harpreet Kaur Grang, ${ }^{2}$ Moshiur Bhuiyan, ${ }^{1}$ P.W.C. Prasad, ${ }^{2}$ Farzana Haque and ${ }^{3}$ A. Elchouemi \\ ${ }^{I}$ School of Computing and Mathematics, Charles Sturt University, Sydney, Australia \\ ${ }^{2}$ Service Consulting, Enterprise Cloud Systems, Sydney, Australia \\ ${ }^{3}$ Colorado State University Global Campus, USA
}

Article history

Received: 04-11-2018

Revised: 04-02-2019

Accepted: 04-03-2019

Corresponding Author:

P.W.C. Prasad

School of Computing and

Mathematics, Charles Sturt

University, Sydney, Australia

Email:cwithana@studygroup.com

\begin{abstract}
The purpose of this research is to identify and address the service delivery issues faced by patients in hospitals including emergency departments. It illustrates the available solutions and determines the one that best fits the solution among service delivery methods in hospitals to provide quick and quality care with better efficiency. There are various software tools that are used to analyse and minimize the patients waiting times. This research examines and discusses the existing practices and findings that are currently being used in the hospital frameworks. It also examines the factors influencing the service delivery and patient satisfaction through different methods and tools to give a clearer picture of the patient flow at the hospitals utilizing a case study approach in the context of hospitals within NSW Australia. This research paper proposes improvement in service delivery model, which potentially improves patient satisfaction as well as increase efficiency for health care service providers to handle huge overcrowding in many hospitals. It potentially creates opportunities for better health care by providing better services on time and in a better quality.
\end{abstract}

Keywords: Hospitals, Emergency Department, Patient Flow, Patient Satisfaction, Service Delivery Innovation, Wait Time, Quality Service

\section{Introduction}

Overcrowding in hospitals is a serious concern that needs considerable and immediate attention as it is increasing the in-hospital mortality rates (Kim et al., 2017). Waiting time or service delivery varies from hospital to hospital; making changes in one hospital cannot solve it. It requires changes in the entire system of the health care. There are several methods, models and software tools that can be used to manage wait times and service delivery in the hospitals. The service delivery modes and length of stay is an important factor in attaining patient satisfaction. Though it has negative impact on the staff's ability to provide quality care. Longer wait times has critical consequences and affects staff's capability to deliver timely care. According to a study (Hoot and Aronsky, 2008), there are numerous factors that contribute to overcrowding in hospitals some of them are: Non-urgent visits, hospital bed shortage, staff shortage, less resources and equipment, frequent-flyer patients, influenza season, treatment delays and negligence etc.

Patient wait time and overcrowding in hospitals is one of the biggest challenges faced by the Health Care
Department. In fact, some of the patients leave without being seen by the doctors (Reinhardt, 2017). This is one of the major confronts faced by the Health Care Department that needs immediate concern. Apart from that, there is also an issue and negligence of the specialty physicians delaying the waiting time of the Emergency department patients. This research opens further opportunities to research to solve the limitations for the safety and the wellbeing of the patients. The research also emphasize on answering questions such as: Who is responsible for the service delay in hospitals? The staff, the management, existing processes, procedures or the system? What are the factors responsible for the waiting time? How the waiting time can be minimized using effective methods and technology? Hence, there is a need to have deep insights in the Health care system.

This study offers enormous benefits not only to the patients but the health care department as well. Some of them are ability to plan effectively ahead, patient flow visualization, maximizing service delivery, effective use of the limited resources, patient satisfaction, profit for organization and quality-care services. This research is could potentially lead to improved, timely and quality care that can improve patient satisfaction. Both current 
and future patients will benefit from the implementation of the innovative service delivery methods.

This paper will investigate patient interaction in availing the hospital services including pre-hospital patient flow, inhospital patient flow, post-hospital patient flow to identify, assess and validate the patient journey. This analysis included value stream mapping to identify the efficiency gains. The work will provide guidance on future work of service improvement in health domain. It has crucial impact on the quality of patient care and patients experience at hospitals (Dods et al., 2013). This study identifies the factors that influences staff performance, patient satisfaction and quality care and addresses the key issues such as workload, efficiency and wait time. The projected outcome of this work is the improved patient journey with an improved and innovative service delivery framework.

This paper is organized as follows: Section I and II presents the introduction and literature review. Section III is ideal patient journey with proposed framework and section IV and $\mathrm{V}$ is implementation and evaluation of model. Conclusion can be found in section VI.

\section{Literature Review}

\section{Service Delivery in Health Care}

Service delivery is an important and critical factor behind patient flow and patient satisfaction in hospitals. Service delivery system represents the quality of service as well as staff's ability to provide timely care (Stewart et al., 2011). It also considers the problems faced by nurses, doctors or physicians in delivering timely and quality care to patients. The results and patient reviews are based on the tools and methods that are currently being used in the hospitals. Apart from that, it also considers the issues and negligence of the specialty physicians delaying the waiting time of the patients at Emergency departments in hospitals.

There are numerous issues with the service delivery system in hospitals. The key issues of service delivery include long wait times, workload on hospital staff, lack of resources and patient satisfaction (Kilcoyne and Dowling, 2008). Providing fast, effective and quality medical service is top priority in hospitals. At present, the time-management and bottom-line pressure has increased in all types of hospitals, as such it is obvious that speed and efficiency are at utmost importance than ever in the hospitals. A study on inefficiency of NSW Hospital states that $83 \%$ of the patients are only seen on time and the waiting time for them is too long. The main issue is to provide:

- The right care: Providing the right care is the most critical factor for patient safety. A patient can be given wrong treatment due to numerous reasons such as failure in communication, failure of pathology test reports, x-ray reports, patient identity failure etc. Providing wrong treatment can have a range of consequences on patient safety from minor to major, resulting in chronic diseases or even death

- At the right time: Providing right care at the right time is another important factor. A patient's safety is directly affected by the timely treatment

- In the right place: Another issue for patient safety is providing the right care at right place with appropriate equipment for diagnosis and treatment.

- With minimum waiting time: There should be minimum wait time in hospitals as per the priority and health condition of patients as it results in higher mortality rate (Rice, 2016)

One of the major factors behind increased wait times of patient flow journey in different hospitals in NSW is the lack of patient's history of medical information to provide immediate and appropriate care. There are several strategies that help in dealing with the issues of service delivery such as providing immediate care as per the health condition of the patients, providing unplanned care for instance in emergency departments, providing alternative options to non-urgent patients to be seen by GP's instead of doctors, timely discharge of patients can have a positive impact on total length of stay, better communication among staff results in providing timely quality care, big data management for providing quick service delivery, informing length of wait times and so on (Zayyan et al., 2017).

\section{Framework, Methods and Tools}

Gul and Guneri (2012) offered a solution called Discrete Event Simulation (DES) model to reduce the patient average Length Of Stay (LOS) and to improve the patient throughput by utilizing the resources efficiently. It was used to ensure that patients demand meets hospital resources. However, the simulation model provides results based on assumed and fixed resources and schedules. Hence, there are further possibilities for improvement to give accurate results in real time environment. Young (2014) proposed modeling and simulation techniques to find new solutions to provide urgent care in hospitals. However, the result shows uncertainty and unintended consequences. In addition, Hayes et al. (2015) proposed a Computer simulation model to improve patient flow but the result shows inaccurate acute comparisons between time periods in a health care system.

According to Iacobucci (2015), "There are no quick fixes for growing waiting times and we need to be prepared to see further breaches of targets in the future". $\mathrm{He}$ offered a solution with planned treatment within a targeted time frame. The goal is to provide timely care to patients to meet the target time frame. However, it affects the quality care and it may result in wrong diagnosis of diseases. According to Rice (2016), TeamStepps tool was introduced and developed by the Agency for Healthcare Research and Quality, which 
promotes teamwork among nurses and doctors to provide patient satisfaction. This tool is very efficient in minimizing wait times and providing quality care. However, there are flaws in the process as patients get inexpert advice of their tests from the nurses instead of seeing doctors. Hence, wait times should be minimized as per the priority and health condition of the patients.

Further, Dinh et al. (2016) offered a solution with Sydney Triage to Admission Risk Tool (START) to predict the in-patient admissions in hospitals. It aims to improve the service flow in hospitals but the overall quality care was compromised. Furthermore, Agarwal et al. (2016) proposed a solution of best management practices to deliver quality health care services. However, it requires continuous changes in the organization as per the patient requirements, which is challenging and difficult to introduce. Lauks et al. (2016) offered Medical Team Evaluation (MTE) as a solution to provide alternative care to patients with quality care. Nonetheless, the result shows improvement in only one ED level, whereas all the other ESI ED level shows increased length of stay of patients. Lanere and Duberman (2017) offered a solution of co-creation strategy with systematic approach to create innovative solutions to health care systems. Though it promotes teamwork and designing strategies but it requires working in an environment with uncertain conditions.

Reinhardt (2017) comes up with another approach of forming a panel of skilled nurses to assess patient's problems by conducting a chart review to develop potential solutions. The solution aims to minimize overcrowding and longer wait times but it does not ensure total quality improvement in the health care system, which is vital for the patient satisfaction. Furthermore, Kim et al. (2017) investigated the impact of service delivery issues in hospitals. They offer a solution that is Fast Track with Wait Time Threshold model (FTW) by combining the existing models of the hospital namely Current ED model (CED) and Fast Track Area model (FTA), leading to tremendous results of cutting wait times by almost $50 \%$. Though, this is an im-provement over the existing solution, the wait time is still unacceptably high. It would, therefore, seem that the combination of models does not offer further possibilities for improvement.

Rezaei et al. (2017) proposed a solution with ideal ED patient journey models to eradicate the problem of overcrowding in hospitals. However, the solution was not appropriate to the real and actual patient flow in hospitals. According to Kim (2017), Centralized patient data is the key to provide appropriate treatment with the help of big data. However, there are security issues with the big data of patient information. Furthermore, Pramanik et al. (2017) proposed 'Big data enabled Smart Healthcare System Framework (BSHSF)' solution to organize patients data and improve the service delivery. Nevertheless, there are security and privacy concerns related to patient information.
Zayyan et al. (2017) conducted a research using quantitative method that helps in determining the factors contributing to wait times and their impact on the patient care. They come up with the solution that wait time is a dependent variable that depends on three independent variables that is Staff, Management and Organizational factors. All three factors (organizational, staff and administrative) have an impact either positively or negatively on the waiting time. Whilst, the solution aims to minimize overcrowding and longer wait times but it is not accurate and it can be improved for delivering better patient care services.

Rajip et al (2017) explored the opportunity for the use of Radio Frequency Identification (RFID) technology in hospital ED to reduce overcrowding. The Australian ED model of care with Triage scale and improved patient journey were studied. The best possible RFID integration was sought and evaluated against health care domain's model of care. Potential indicators of suitability were ED length of stay, ED wait times. Ambulance diversions were studied and contrasted from the start of the patient journey to the end of the patient's treatment cycle, to find opportunities for the implementation of RFID technology.

\section{Current Framework}

Managing patient flow in hospitals is a complex process that requires coordinated approach between departments, services and people to provide safe, effective and timely care according to patient needs (Iacobucci, 2015). The Fig. 1 shows the key factors impacting on the patient flow journey in hospitals.

It includes 3 critical factors that is:

\section{Pre-Hospital Patient Flow}

In pre-hospital, there is Hospital In The Home (HITH) service that provides an acute hospital substitution for patients where care is provided at home by General Practitioners, doctors or nurses. Key factors under pre-hospital patient flow are potentially avoidable admissions, better alternative to hospital care and improved chronic disease management.

\section{In-Hospital Patient Flow}

Hospitals and emergency departments come under inhospital patient flow. There are numerous factors affecting the in-patient flow such as artificial scheduling of resources and staff and bed turnover time to improve capacity management in hospitals. There are further two main categories that are Inflow and Outflow. Inflow covers the Emergency Department (ED) issues such as overcrowding, wait time, lack of bed availability that further leads to access block for ED patients. Under outflow, the key factors include delayed discharge resulting in complex admission process; delays in care processing that is wait time to be seen by doctors. It requires better communication and coordination among staff members to overcome the issue. 


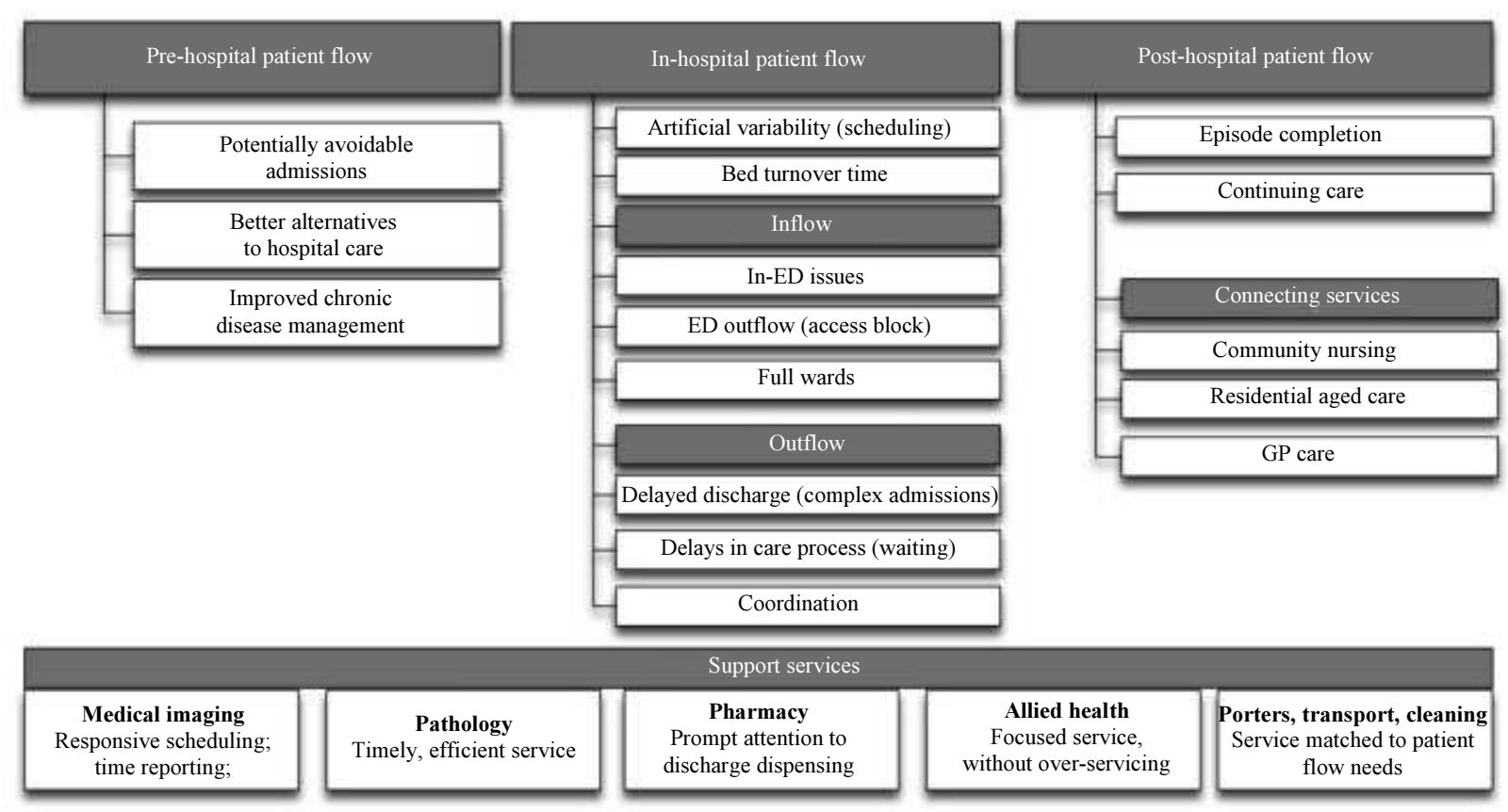

Fig. 1: Key factors impacting on patient flow journey (PFS, 2017)

\section{Post-Hospital Patient Flow}

Key factors in post-hospital include patient discharge from the hospital by either episode completion or continuing care. GP care, community nursing or residential aged care services come under post-hospital patient flow.

The following diagram represents the patient flow journey in hospitals that begins with a planned visit that is walk-in or unplanned visit that is emergency visit.

In a planned visit, a patient has to go through registration process as soon as he walks in the hospital that is followed by a wait time to be seen by a nurse at arrival, which further goes to the assessment area. In the assessment area, the nurses will assess the urgency of illness on to the scale of 1 to 5 . Based on the order of urgency, the patients are further given their wait times to be seen by doctors. If the doctors direct the patient for any tests such as x-rays, blood test etcetera they have to wait for their tests to be done as well as for the test results. On the basis of test results, patients are either directed to their homes or admitted in the hospitals. However, non-urgent patients have maximum wait time to be seen by the doctors. On the other hand, emergency department begins with the assessment of illness by nursing staff on to the scale of 1 to 5 , to determine the priority of treatment. The registration process is followed only if the patient's condition is less urgent, otherwise the patient's with 'high priority' health condition are sent immediately to the treatment zone without any delay.

Different key factors are combined to represent the current framework of hospitals in NSW that is shown in following Fig. 3. The patient flow follows the same stages from walk-in to walkout for planned as well as unplanned visits as depicted in Fig. 2, 'Patient flow journey'. Though, the registration process for planned visit is followed by a wait time that is a limitation in the current framework as this wait time can be minimized and eradicated completely. It only increases the total length of stay and requires more staff and resources.

In Fig. 3, the limitation is highlighted with red color, though the critical factors are highlighted with blue color.

The following 7 key elements are critical for ideal patient flow journey in hospitals according to NSW Health Government (PFS, 2017):

- Demand and capacity planning: It is an approach to manage workload in most efficient manner. It matches the patient demand with available staff and resources

- Variation management: It controls and manages the internal variations within the hospitals for smooth and better outcomes with coordinated approach

- Demand escalation: It helps in managing patient demand with available re-sources before the capacity becomes an issue such as bed management

- Standardized practice: It is an approach of benchmarking the expectations from staff, managers and health care facility. Hence, it is possible to identify the problems that do not meet the standardized criteria and thus propose the expected solutions 
- Care coordination: It is an approach of managing patients from the admissions process to the discharge. It helps in minimizing the wait times throughout the patient journey by effective communication process among staff members

- Governance: Governance structure must exist in every organization for the effective implementation and sustainability of business process. It helps focusing staff to make decisions based on the patient centered care

- $\quad$ Quality: It focuses on providing the quality care to the patients and improving the patient satisfaction throughout the journey in hospitals with the help of standardized practices

\section{Justification}

The flow of patients in hospitals is increasing day by day, with each patient having their own needs to be met. Maintenance and organization of the flow of patients along with service delivered to them are essential. Service Delivery in such an environment is a tough job for management team and medical staff. The strength to service delivery comes from within the structure of the department, the management team, the business process and framework of hospitals. The current patient flow include the key factors such as providing alternatives to patients to hospital care, improved chronic disease management, bed turnover time, continuing care, different support services and so on.

\section{Issues}

The patient flow journey lacks the patient's history or record of medical in-formation, which is a major factor behind increased wait times in different hospitals (Pramanik et al., 2017). According to Kim (2017), Centralized patient data is the key to provide appropriate treatment with the help of big data. The current model requires a Centralized health care system to manage big data and thus providing quick and timely services to patients.

Existing solution to the issues: DIGITAL HEALTH: 'My Health Record' in Australia. It maintains patient's health information online at one place that includes patient's treatment details, chronic diseases, allergies, pathology reports and medicine details etcetera that can be accessed by doctors, GP's during emergencies (My Health Record, 2017).

Using digital health system GP's, physicians or doctors upload all the patients' medical history at one place that can be retrieved during emergencies throughout the NSW in all the registered clinics as well as hospitals. It saves a great deal of patient's and hospitals wait time particularly in emergency cases. It provides access to patient information recorded by GPs and physicians that helps in understanding of patient's medical history and thus provides quick diagnosis. It also helps in minimizing total length of stay and makes effective use of hospital staff and resources. It improves patient satisfaction and reduces mortality rate.

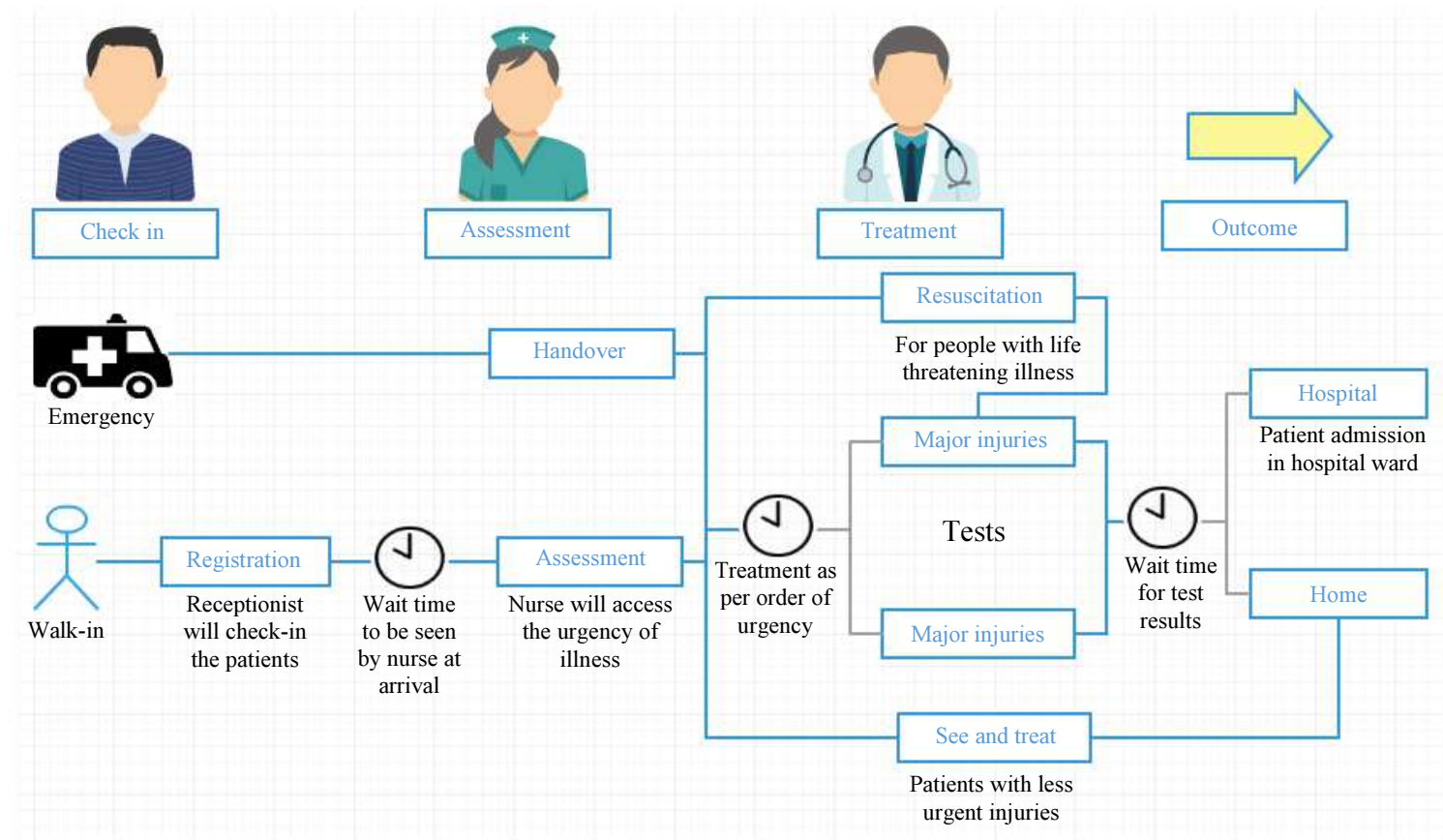

Fig. 2: Patient flow journey in hospitals 


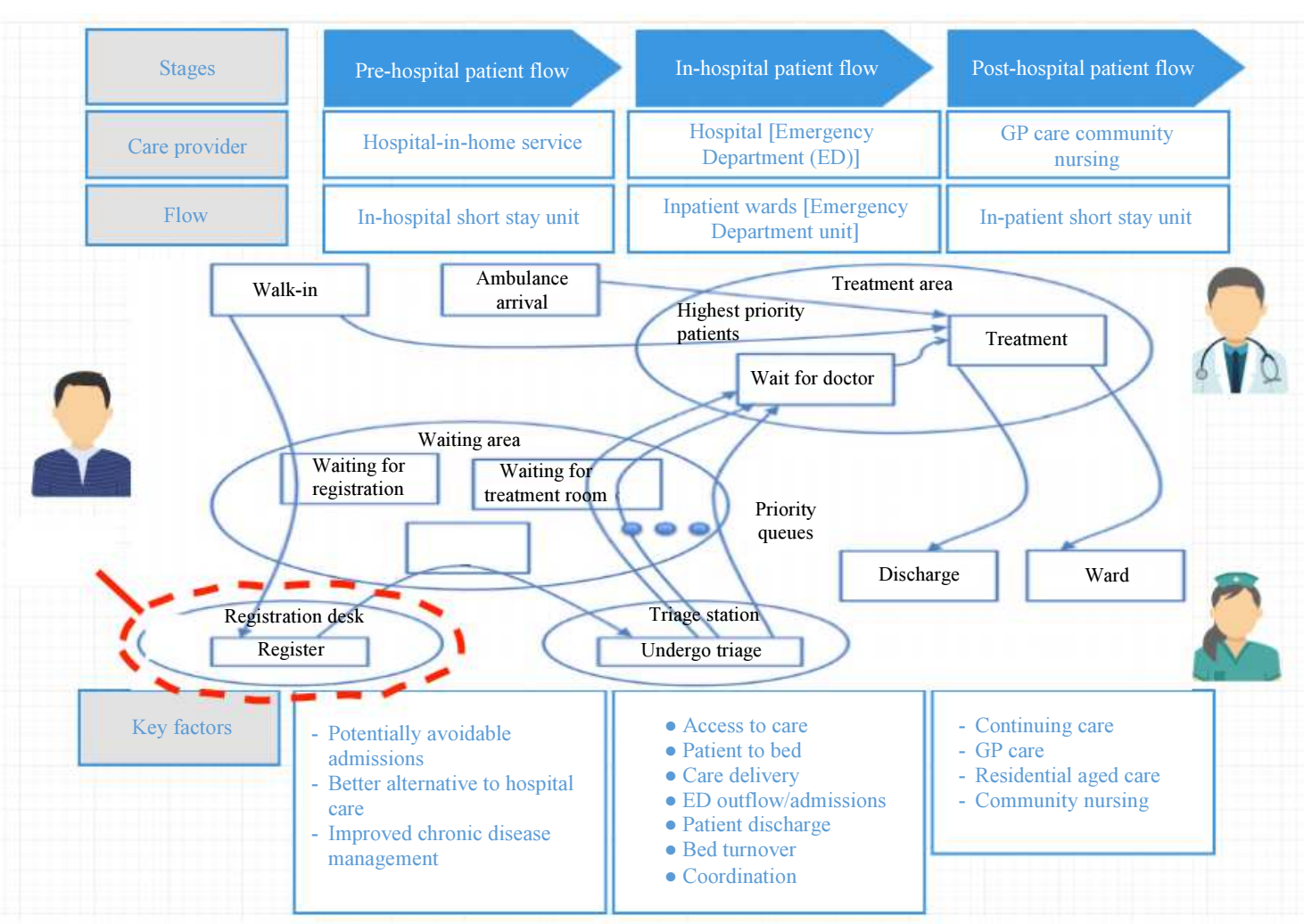

Fig. 3: Current framework of patient flow
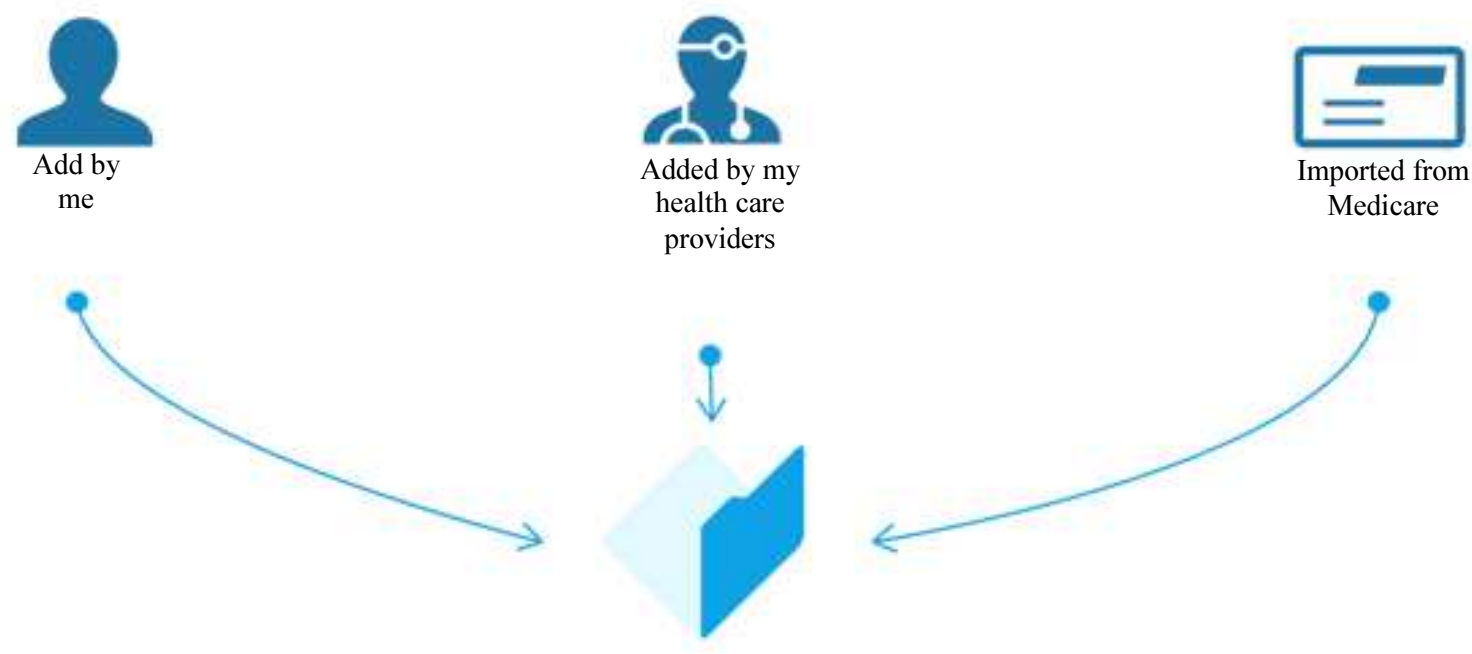

Fig. 4: Australian digital health agency

\section{An Ideal Patient Journey}

An ideal patient journey includes everything from patient's check-in to checkout in hospitals. It provides the timely care along with patient satisfaction. Ideal patient flow is also critical for improving efficiency and experience of patients (Rezaei et al., 2017).

\section{Proposed Framework}

'Current framework of patient flow' in Fig. 3 illustrates a planned visit in hospital constitute of total 4 wait times from registration process to the discharge of the patients that further contributes to the total length of stay. These are wait for registration, wait for triage, wait 
for treatment room and Wait for doctor. Figure 4 details process of digital health agency.

The proposed framework (illustrated in Fig. 5) is an improved version of current framework that helps in rectifying the issues of current framework. It helps in minimizing the wait time at the entry point that is 'Wait for registration process' by introducing Digital health service and kiosk service.

The proposed framework shows the critical stages in patient flow namely pre-hospital, in-hospital and posthospital flow. Under pre-hospital patient flow, there is Hospital in-home service that provides health care services at home to help prevent the patient admissions and thus overcrowding in hospitals. In-hospital is the emergency or planned visit in the hospitals. Post-hospital includes follow-up patient care after discharge from hospital that includes GP care or community nursing. Patient flow consists of numerous stages from the walkin until discharge from the hospitals. There is long wait time in the process at different stages to see the doctor until unless there is emergency that needs immediate and critical care. From the moment patient walk-in to the hospital, he has to wait for the registration process, while that can be minimized with the health kiosk service in hospitals that allows patients to register themselves as soon as they walk-in without any wait.

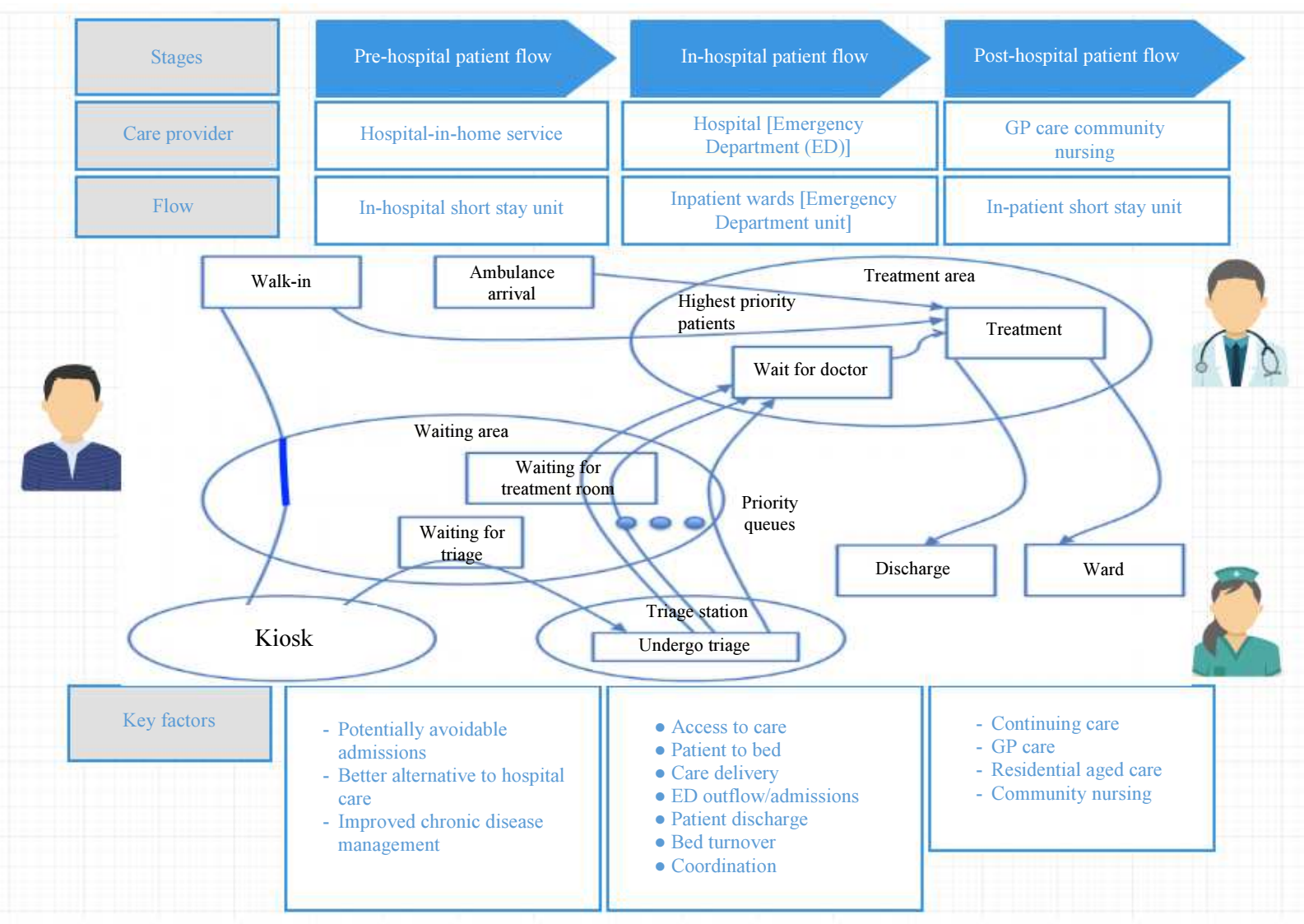

Fig. 5: Proposed framework

Table 1: Australasian triage scale

\begin{tabular}{llll}
\hline $\begin{array}{l}\text { Australasian triage } \\
\text { scale category }\end{array}$ & $\begin{array}{l}\text { Treatment acuity (Maximum waiting } \\
\text { time for medical assessment and treatment) }\end{array}$ & $\begin{array}{l}\text { Performance } \\
\text { indication threshold }\end{array}$ & $\begin{array}{l}\text { Triage } \\
\text { category type }\end{array}$ \\
\hline ATS 1 & Immediate & $100 \%$ & Resuscitation \\
ATS 2 & $10 \mathrm{~min}$ & $80 \%$ & Emergency \\
ATS 3 & $30 \mathrm{~min}$ & $75 \%$ & Urgent \\
ATS 4 & $60 \mathrm{~min}$ & $70 \%$ & Less-Urgent \\
ATS 5 & $120 \mathrm{~min}$ & $70 \%$ & Non-Urgent \\
\hline
\end{tabular}




\section{Key Areas}

According to NSW health government (PFS, 2017), there are numerous factors to help smooth the patient flow that is bed management, communication which is critical factor among hospital staff, timely care delivery, timely patient discharge standardizing clinical and administrative process and identifying and removing bottlenecks in the system.

Different key areas in the proposed framework include patient walk-in, kiosk registration, triage station, resuscitation (see doctor without any wait), emergency and urgent care (goes to the treatment area), less urgent and non-urgent care (goes to the waiting area).

\section{Australasian Triage Scale (ATS)}

On arrival, patients are categorized on to a scale of 1 to 5 (refer to Table 1) to know the level of urgency of their health condition and thus treated as per the following criteria.

\section{Implementation of the Model}

The implementation of the proposed model of patient flow with the health kiosk is a self-service solution for patients that not only minimize wait time but also provide enormous features.

\section{An Innovative Approach}

Health kiosk is the next generation health service in hospitals. It provides following advantages:

- Self Check-in: It provides self check-in option for patients instead of filling paperwork during registration process. This information can be saved and further retrieved during each visit of patient, which ultimately saves time

- Low cost: It is not only beneficial for patients but also for health care facilities. It reduces cost, data entry errors and burden of administrative work and provides more time for patient care

- Virtual triage kiosk: It helps in providing services of triage nurse by giving advise on the health condition of the patients. It includes asking symptoms, accessing current health condition, checking vision, measuring height and weight

- Way finding: It is also useful in providing information in hospitals using map such as parking information, ward information

- $\quad$ Easy payment: Patients prescription payments are easy to pay via kiosk
- $\quad$ Requires fewer employees: Effective use of staff and resources

- Live diagnostic processes: It also connects patients with live healthcare professionals for diagnostic processes

\section{Features}

Health kiosk covers digital health and patient information. As shown in Fig. 6, there are 3 main features of health kiosk:

\section{Queue and Information System}

It displays real time information that helps in providing actual wait time to the patients. It results in reduced waste time and shorter length of stay.

\section{Automated Front Desk System}

It helps patients performing payment procedure themselves by providing guidelines. Thus, fewer staff is required for billing and payment purposes.

\section{Automatic Returning Patient}

It helps in completing registration process quickly at reception area to minimize wait time in patient flow. It also keeps record of the number of visits along with patient history, making the process smooth and quick for patients as well as hospital staff.

\section{Evaluation of Implementation}

The evaluation process explains the outcomes and challenges of proposed framework in a working environment with 'Health kiosk' service and 'My health record' from Australian digital health agency.

The positive outcome of this solution enhances the quality of patient care and improves staff's ability to provide timely care by minimizing wait time at initial stage. The proposed model is significantly beneficial for reducing wait time in hospitals. In addition, it is also easy to operate, as its functionality is user friendly. It can verify the patients by login and password or by asking them to scan their Medicare card. Hence, it also provides security. It reduces long wait time in the process at initial stage to see the doctor. Depending on the health condition of the patients they are further categorized under the scale of ATS to wait see the doctors.

On the other hand, using 'My health record', GP's, physicians or hospitals can view and upload all the patients' medical history at one place that can be retrieved during emergencies throughout the NSW in all the registered clinics as well as hospitals. It saves a great deal of patient's and hospitals wait time particularly in emergency cases. 


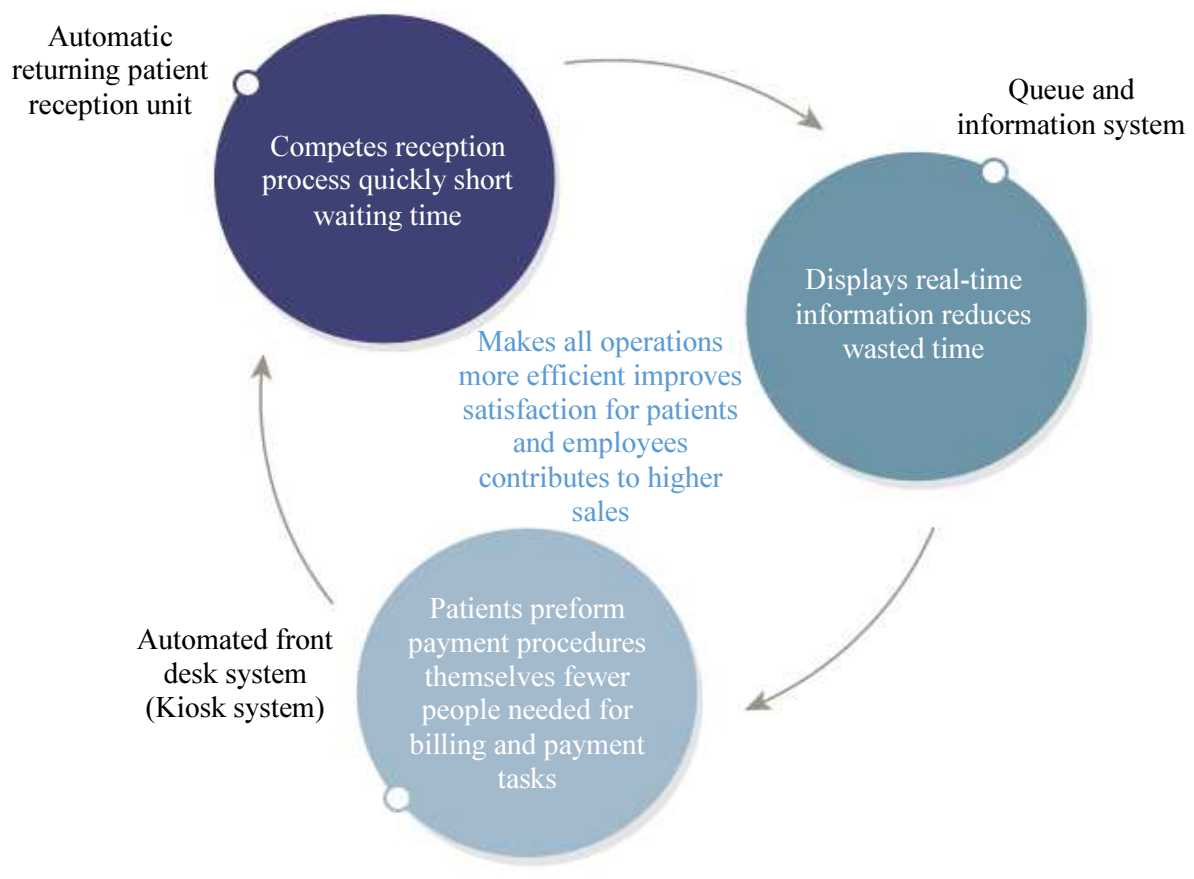

Fig. 6: Features of kiosk

\section{Conclusion}

The expected outcome of this solution is to propose an improved framework with innovative service delivery methods to enhance the quality of patient care and improve the staff's ability to provide timely care. The projected outcome will benefit the hospitals get insight into managing the health care services. The current solution helps in minimizing the barrier between patient satisfaction and quality health care services. It include the key factors such as providing alternatives to patients to hospital care, improved chronic disease management, bed turnover time, continuing care and different support services. It also examines the factors that influence the total length of stay of patients in hospitals.

The proposed framework is categorized by three critical stages in patient flow namely pre-hospital, inhospital and post-hospital flow. Under pre-hospital patient flow, there is Hospital in-home service that provides health care services at home to help prevent the patient admissions and thus overcrowding in hospitals. In-hospital is the emergency or planned visit in the hospitals. Post-hospital includes follow-up patient care after discharge from hospital that includes GP care or community nursing. This framework provides guidance to staff on providing quick and quality care to the patients. It is recommended that the patients should monitor their waiting time and services in the hospitals. The research has successfully evaluated the current frame-work and proposed framework in terms of the key elements such as wait-time, total length of stay, workload, bed management and promoting communication among staff. The reduced wait time is the most significant success of the proposed concept.

Overall, the proposed framework identifies several approaches for providing timely care in hospitals with different factors such as staff management, bed management, scheduling surgeries, quality care and so on. Moreover, this study determines that the innovative service delivery methods or tools are superior to the traditional patient flow management tools in hospitals. Although this concept is useful and harmless, but health domain is a critical sector putting life at risk, hence implementation of this project in future should be started from small and controllable area, to look into actual results in all NSW hospitals. Furthermore, the future research should pay attention to the issue of providing timely quality care within hospitals rather than focusing on only wait time because wait time is only one aspect of the problem. At last, ethical issues will be considered to mitigate the risk of privacy and confidentiality breach.

\section{Author's Contributions}

Harpreet Kaur Grang: Harpreet is the lead author; her contribution includes literature review, establishing principles of service design, organisational design and development of the framework.

Moshiur Bhuiyan: Supervised this work including providing guidance, direction and contribution to the methodology. 
P.W.C. Prasad: Supervised/worked closely with Harpreet Grang during the analysis, design and experiment phases.

Farzana Haque: Contributed to the refinement of the methodology and assisted in final review.

A. Elchouemi: Contributed in the technical content, addressed the corrections, checked the final review and provide the required funding for the paper.

\section{Ethics}

This article is original and contains unpublished material. The corresponding author confirms that all of the other authors have read and approved the manuscript and no ethical issues involved.

\section{References}

Agarwal, R., R. Green, N. Agarwal and K. Randhawa, 2016. Management practices in Australian healthcare: Can NSW public hospitals do better? J. Health Organiz. Manage., 30: 331-353.

Dinh, M.M., B.R. Saartje, K.J. Bein, K. Rogers and D. Muscatello et al., 2016. The Sydney Triage to Admission Risk Tool (START) to predict emergency department disposition: A derivation and internal validation study using retrospective statewide data from New South Wales, Australia. BMC Emergency Med. DOI: 10.1186/s12873-016-0111-4

Dods, S., J. Boyle, S. Khanna, J. O'Dwyer and D. Sier et al., 2013. Evidence driven strategies for meeting hospital performance targets - The value of patient flow modelling. CSIRO.

Gul, M. and A.F. Guneri, 2012. A computer simulation model to reduce patient length of stay and to improve resource utilization rate in an emergency department service system. Int. J. Industrial Eng., 19: 221-231

Hayes, K.J., K. Eljiz, A. Dadich, J. Fitzgerald and T. Sloan, 2015. Trialability, observa-bility and risk reduction accelerating individual innovation adoption decisions. J. Health Organiz. Manage., 29: 271-294.

Hoot, N. and D. Aronsky, 2008. Systematic review of emergency department crowding: Causes, effects and solutions. Annals Emergency Med., 52: 126-136. DOI: 10.1016/j.annemergmed.2008.03.014

Iacobucci, G., 2015. Reasons for longer hospital waiting times need to be understood, says Nuffield trust. Brit. Med. J. DOI: 10.1136/bmj.h1411

Kilcoyne, M. and M. Dowling, 2008. Working in an overcrowded accident and emergency department: Nurses' narratives. Austral. J. Adv. Nurs.
Kim, B., T. Delbridge and D. Kendrick, 2017. Adjusting patients streaming initiated by a wait time threshold in emergency department for minimizing opportunity cost. Int. J. Health Care Quality Assurance, 30: 516-527.

Kim, J., 2017. Big data, health informatics and the future of cardiovascular medicine. J. Am. College Cardiol., 69: 899-902. DOI: 10.1016/j.jacc.2017.01.006

Lanere, J.B. and T. Duberman, 2017. Co-creating strategy. Trustee, 70: 5-5.

Lauks, J., B. Mramor, K. Baumgartl, H. Maier and C.H. Nickel et al., 2016. Medical team evaluation: Effect on emergency department waiting time and length of stay. PLoS One. DOI: 10.1371 /journal.pone. 015372

My Health Record, 2017. My Health Record | What is My Health Record? Myhealthrecord.gov.au.

PFS, 2017. Patient Flow Systems. Health.nsw.gov.au.

Pramanik, M., R. Lau, H. Demirkan and M. Azad, 2017. Smart health: Big data enabled health paradigm within smart cities. Expert Syst. Applic., 87: 370-383.

Reinhardt, M., 2017. A systematic approach to evaluation of performance deficiencies in ED triage. J. Emergency Nurs., 43: 329-332.

Rajip Raj Thapa, Moshiur Bhuiyan, Aneesh Krishna, P. W. C. Prasad: Use of Radio Frequency Identification Technology in Reducing Overcrowding at Hospital Emergency Departments. ISD 2017

Rezaei, F., M. Yarmohammadian, A. Haghshenas and N. Tavakoli, 2017. Overcrowding in emergency departments: A review of strategies to decrease future challenges. J. Res. Med. Sci., 22: 23-23. DOI: $10.4103 / 1735-1995.200277$

Rice, S., 2016. Cutting emergency department wait times as patient volumes rise. Modern Healthcare, 46: 28-28.

Stewart, J., S. Lohoar and D. Higgins, 2011. Effective practices for service delivery and coordination in Indigenous communities. Australian Institute of Health and Welfare.

Young, T., 2014. Test drive the new care model. Health Service J., 124: 20-21.

Zayyan, O., M. AlBarakati, A. Aziz, H. Al-Borie and A. Khalaf, 2017. Factors influencing waiting time as key of patient satisfaction in the Emergency Department in King Fahd Armed Forces Hospital, Saudi Arabia. Int. J. Bus. Manage., 12: 79-79. DOI: 10.5539/ijbm.v12n5p79 